\title{
TRIM25 in the Regulation of the Antiviral Innate Immunity
}

\author{
María Martín-Vicente', Luz M. Medrano', Salvador Resino', Adolfo García-Sastre ${ }^{2,3,4}$ \\ and Isidoro Martínez ${ }^{1 *}$ \\ 1 Unidad de Infección Viral e Inmunidad, Centro Nacional de Microbiología, Instituto de Salud Carlos III, Madrid, Spain, \\ ${ }^{2}$ Department of Microbiology, Icahn School of Medicine at Mount Sinai, New York, NY, United States, ${ }^{3}$ Global Health and \\ Emerging Pathogens Institute, Icahn School of Medicine at Mount Sinai, New York, NY, United States, ${ }^{4}$ Department of \\ Medicine, Division of Infectious Diseases, Icahn School of Medicine at Mount Sinai, New York, NY, United States
}

\section{OPEN ACCESS}

Edited by: Thomas A. Kufer, University of Hohenheim, Germany

Reviewed by: Ashley Mansell, Hudson Institute of Medical Research, Australia Isabelle Vergne, Centre National de la Recherche Scientifique (CNRS), France

${ }^{*}$ Correspondence: Isidoro Martínez imago@isciii.es

Specialty section: This article was submitted to Molecular Innate Immunity, a section of the journal

Frontiers in Immunology

Received: 06 July 2017 Accepted: 07 September 2017 Published: 22 September 2017

Citation: Martín-Vicente M, Medrano LM, Resino S, García-Sastre A and Martínez I (2017) TRIM25 in the Regulation of the Antiviral Innate Immunity.

Front. Immunol. 8:1187. doi: 10.3389/fimmu.2017.01187
TRIM25 is an E3 ubiquitin ligase enzyme that is involved in various cellular processes, including regulation of the innate immune response against viruses. TRIM25-mediated ubiquitination of the cytosolic pattern recognition receptor RIG-I is an essential step for initiation of the intracellular antiviral response and has been thoroughly documented. In recent years, however, additional roles of TRIM25 in early innate immunity are emerging, including negative regulation of RIG-I, activation of the melanoma differentiationassociated protein 5-mitochondrial antiviral signaling protein-TRAF6 antiviral axis and modulation of p53 levels and activity. In addition, the ability of TRIM25 to bind RNA may uncover new mechanisms by which this molecule regulates intracellular signaling and/ or RNA virus replication.

Keywords: TRIM25, innate immunity, ubiquitination, virus, E3 ubiquitin ligase

\section{INTRODUCTION}

The innate immune response is the first line of defense against invading pathogens. At the cellular level, this response is stimulated by several cellular "pattern recognition receptors" (PRRs) that recognize microbial-specific molecules termed "pathogen-associated molecular patterns" (PAMPs). Bacterial PAMPs include lipopolysaccharide (LPS), flagellin, peptidoglycan, and cyclic dinucleotides, among others. Although viral proteins are able to stimulate specific PRRs, the main viral PAMPs are nucleic acids, including double-stranded RNA (dsRNA), uncapped single-stranded RNA, and viral DNA (1-3). Two main groups of PRRs that recognize virus-derived nucleic acids have been described according to their location: (1) membrane-spanning toll-like receptors (TLRs), which detect viral RNA or DNA in endosomes and (2) cytoplasmic sensors, including RIG-I-like receptors (RLRs), NOD-like receptors (NLRs), which recognize cytoplasmic viral RNA, and a group of structurally unrelated intracellular viral DNA sensors (1) (Figure 1). Binding of PAMPs to PRRs leads to the activation of intracellular signaling pathways that produce type I interferons and inflammatory cytokines (4). These pathways converge at the level of several kinases of the inhibitor of nuclear factor kappa-B [Ik $\beta$ ] kinase (IKK) family: the canonical complex composed of IKK $\alpha$, IKK $\beta$ and the regulatory subunit IKK $\gamma / \mathrm{NEMO}$, and the non-canonical IKK $\varepsilon$ and TANK-binding kinase-1 (TBK1) (Figure 1). The IKK $\alpha / \beta / \gamma$ complex activates nuclear factor kappa B (NF- $\mathrm{KB}$ ), while TBK1/IKK $\varepsilon$ activates IFN-regulatory factors 3 and 7 (IRF3/7) (5, 6). In addition, PRRs trigger phosphorylation of several mitogen-activated protein kinases (MAPK), which leads to activation of activator protein-1 (AP-1). Upon translocation into the nucleus, the transcription factors NF- $\mathrm{kB}$, IRF3/7, and AP-1 induce the expression of IFNs and other cytokines and antiviral molecules (7) 


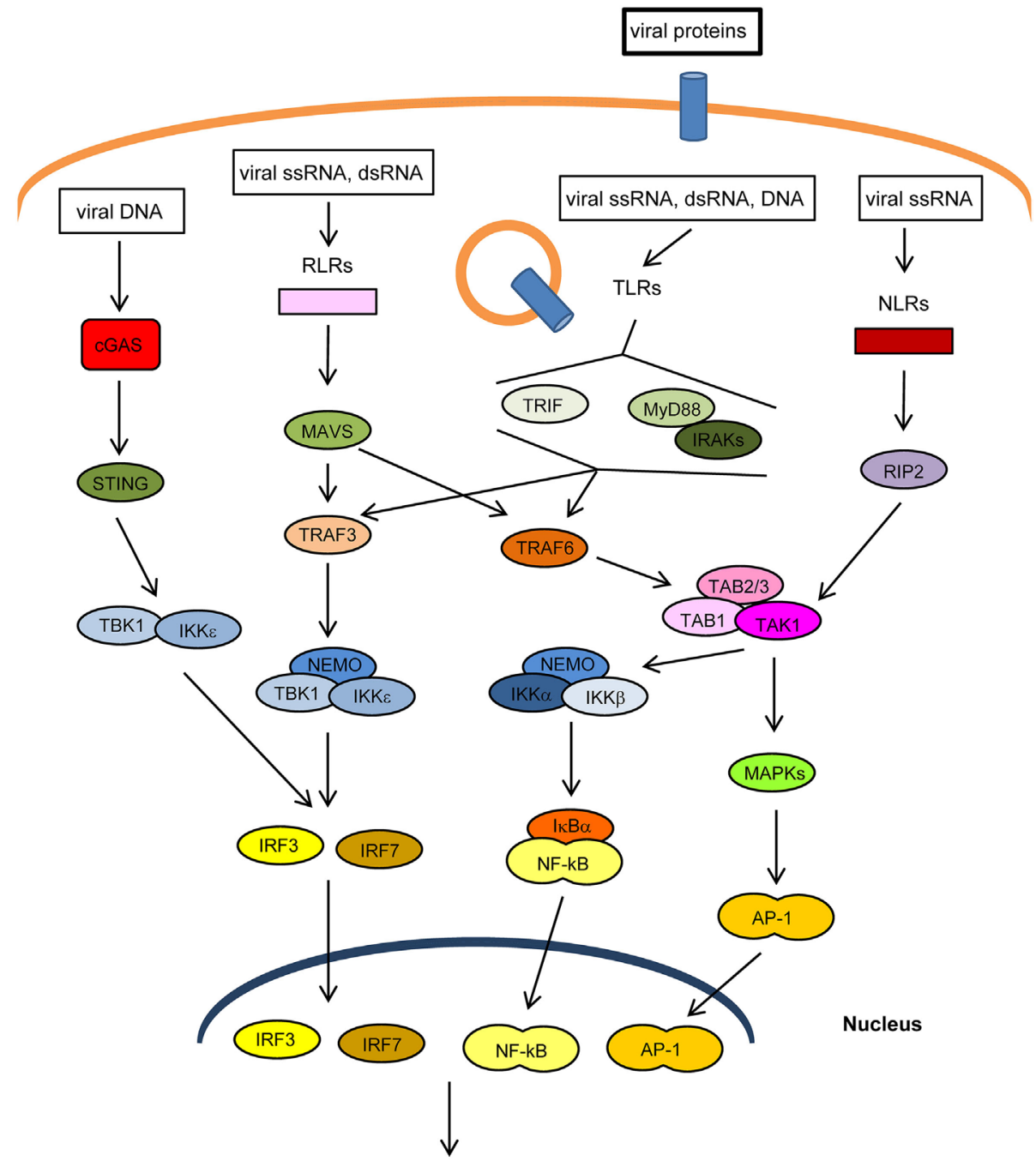

Antiviral response

FIGURE 1 | Main pathways of antiviral innate immunity. After recognition of pathogen-associated molecular patterns (PAMPs) by pattern recognition receptors (PRRs) [cGAS, RIG-I-like receptors (RLRs), toll-like receptors (TLRs), NOD-like receptors (NLRs)], the signal is propagated through adaptor proteins (STING, MAVS, MyD88, TRIF) to members of the TRAF ubiquitin E3 ligase family (TRAF3/6) and to kinase complexes (IKK $\alpha / \beta / \gamma$, TBK1/IKK $\varepsilon$, TAK1/TAB $1 / 2 / 3$, MAPKs), which trigger the activation of IRF3/7, NF-кB and AP-1 transcription factors and the expression of IFN-I, proinflammatory cytokines, and other antiviral genes. The activity or stability of most of these proteins is regulated by ubiquitination/deubiquitination processes.

(Figure 1). These molecules not only inhibit viral replication, assembly, and spread but also play a crucial role in activating the adaptive immune response (8).

The innate immune response is crucial for limiting viral infections, but it has to be tightly regulated to avoid immune-mediated tissue damage, excessive inflammation, and auto-immunity (9-11). In this regard, the complex interactions between molecules of intracellular signaling networks leading to the antiviral response are regulated by various mechanisms, of which posttranslational protein modification is one of the most relevant. It has been known for a long time that phosphorylation plays a crucial role in the regulation of such networks but, in recent years, reversible conjugation to ubiquitin and ubiquitin-like proteins has emerged as an additional and central mechanism regulating intracellular signaling (12-16).

\section{UBIQUITINATION/DEUBIQUITINATION PROCESSES ADJUST THE INNATE IMMUNE RESPONSE}

Ubiquitin is a 76 amino acid $(8.5 \mathrm{kDa})$ protein that can be covalently ligated to lysine residues of a target protein through its conserved C-terminal di-glycine motif (17). Ubiquitination is a three-step enzymatic process involving three enzymes with distinct functions: E1 activating, E2 conjugating, and 

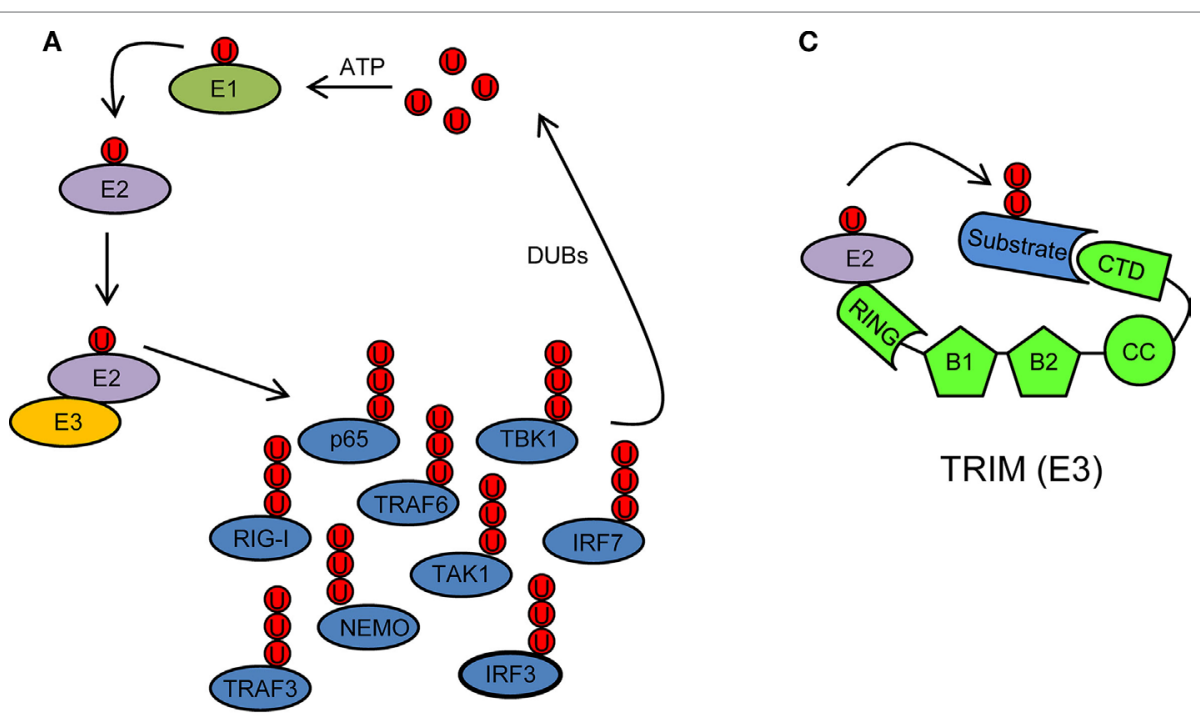

$\operatorname{TRIM}(\mathrm{E} 3)$

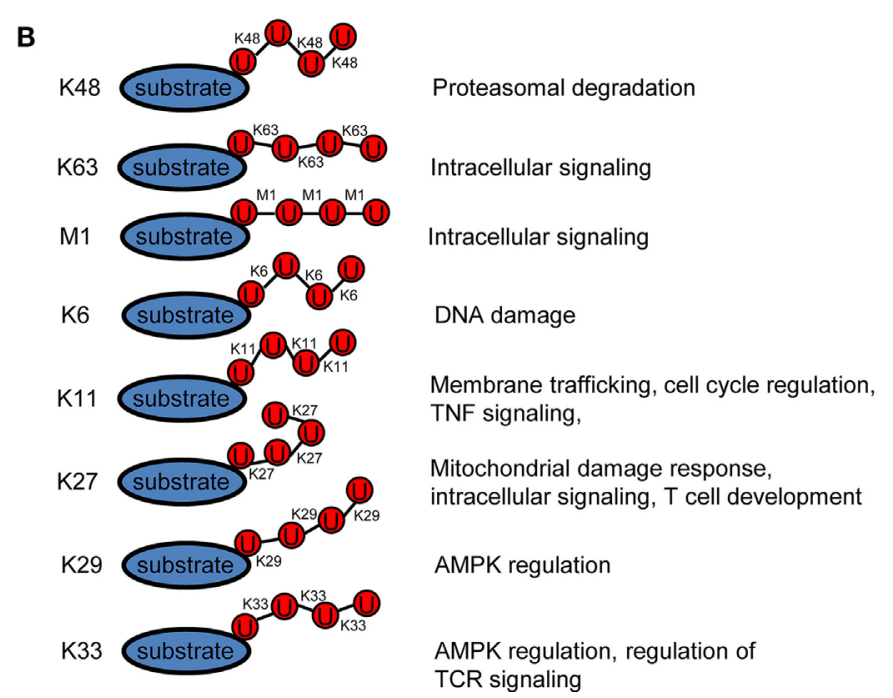

FIGURE 2 | Ubiquitination/deubiquitination regulates intracellular immune pathways. (A) Ubiquitination is a three-step enzymatic process by which ubiquitin is covalently attached to target proteins, including ubiquitin itself. This process is reversed by deubiquitinases (DUBs). The activity of several molecules (p65, RIG-I, TRAF3/6, NEMO, IRF3/7, etc.) participating in intracellular immune signaling pathways is regulated by ubiquitination/deubiquitination modifications. E1, ubiquitin activating enzyme; E2, ubiquitin conjugating enzyme; E3, ubiquitin ligase. (B) Ubiquitin linkage types and their functional roles. Different ubiquitin linkages led to different conformations of ubiquitin chains that are recognized by proteins containing ubiquitin-binding domains. (C) Schematic representation of a tripartite motif (TRIM) molecule, including the conserved N-terminal TRIM [really interesting new gene (RING), B box, and coiled-coil (CC) domains] and a C-terminal variable domain (CTD). Ubiquitin-loaded E2 and substrate are recognized by the RING and CTD domains of TRIM, respectively. This brings both molecules in close proximity and facilitates substrate ubiquitination. Ubiquitin molecules in $(\mathbf{A}-\mathbf{C})$ are represented by a $U$ inside a red circle. The number of $U$ s does not represent the actual level of ubiquitination.

E3 ligating (Figure 2A). The first step is the formation of a thioester linkage between ubiquitin and the E1. In the next step, ubiquitin is transferred from E1 to the active-site cysteine of E2. Finally, E3 assists the formation of an isopeptide bond between the $\mathrm{C}$-terminal glycine of ubiquitin and a lysine residue of a target protein (18-21). Ubiquitin itself can be ubiquitinated and form polyubiquitin chains (polyubiquitination). Ubiquitin contains seven lysine residues (K6, K11, K27, K29, K33, K48, and K63) on which diverse chain types of polyubiquitin can be assembled (20) (Figure 2B). In addition, ubiquitin chains can also be linked in a linear (M1) fashion by attachment of the C-terminal glycine of an ubiquitin to the N-terminal methionine of another ubiquitin, resulting in a head-to-tail polyubiquitination (22) (Figure 2B). Different ubiquitin linkages fulfill different functions (Figure 2B). K48- and K63linked polyubiquitin chains are the best characterized. K48 linkage targets proteins for proteasomal degradation, whereas K63 and M1 linkages regulate intracellular immune signals $(14-16,23,24)$. The other linkage types are referred as "atypical” and have not been studied in much detail (25). Each chain 
type has a different three-dimensional conformation that is recognized specifically by ubiquitin-binding proteins (UBPs). This recognition is essential for the transmission of intracellular signaling (26-28). Recently, it has been reported that in addition to the above described covalently attached polyubiquitin chains, unanchored polyubiquitin chains also contribute to the activation of intracellular pathways leading to the onset of the antiviral response $(29,30)$.

Ubiquitination is reversed by deubiquitinases (DUBs) that detach ubiquitin from the substrate (Figure 2A). This usually leads to termination of immune signaling. In this way, ubiquitination/deubiquitination processes dynamically regulate the early innate immune response and prevent immune-mediated host damage (13).

\section{TRIPARTITE MOTIF (TRIM) PROTEINS ARE E3 UBIQUITIN LIGASES}

Tripartite motif proteins have E3 ubiquitin ligase activity and form a large family with over 70 members in humans. Their name is derived from the fact that they share three conserved N-terminal domains: a really interesting new gene (RING) domain, one or two B-Boxes (B1/B2) and a coiled-coil (CC) domain. By contrast, the $\mathrm{C}$-terminal region is of variable composition $(31,32)$ (Figure 2 C). The RING and B-box domains are both cysteine-histidine-rich domains that bind zinc atoms. The RING domain recognizes the ubiquitin-loaded E2 conjugating enzyme and promotes ubiquitin conjugation to target proteins (32-35) (Figure 2C). Based on conserved structural features with the RING domain, it has been suggested that the B-boxes could also contribute to E3 ubiquitin ligase activity of TRIM proteins $(36,37)$. In some TRIMs, B-box domains mediate selfassociation, which may be important for TRIM oligomerization (38-40). The CC domain is necessary for dimerization of TRIM ligases (41). Finally, the variable C-terminal domain may mediate interaction with specific substrates (Figure 2C). The most common C-terminal TRIM domain is the PRY-SPRY or B30.2 domain, which has been proposed to be involved in protein-protein interactions and/or RNA binding (42).

Tripartite motif E3 ligases regulate many cellular processes, including development, cell growth, differentiation, cancer, and innate immune response $(32,43-45)$. Several TRIMs have been reported to exhibit antiviral activity either directly or through regulation of antiviral cell signaling (45-53).

\section{TRIM25 REGULATES INTRACELLULAR SIGNALING}

TRIM25 is a type I and type II IFN-inducible E3 ligase (54) that was first identified as an "estrogen-responsive finger protein" (EFP) (55). It is composed of a RING domain, two B-boxes domains, a CC dimerization domain and a C-terminal SPRY domain (Figure 3).

TRIM25 is involved in numerous cellular processes, such as development, cancer, and innate immunity (56). Regulation of RIG-I signaling by K63-linked polyubiquitination is one of the best-characterized roles of TRIM25 (57). Recognition of viral
RNA by RIG-I exposes its 2CARD domain for binding to the C-terminal SPRY domain of TRIM25 (Figure 3). The RING E3 ligase activity of TRIM25 then conjugates K63-polyubiquitin chains to residues K99, K169, K172, K181, K190, and K193 of RIG-I (57-59) (Figure 3). The K63-linked ubiquitin chains on RIG-I promotes its interaction with "mitochondrial antiviral signaling protein” (MAVS, also known as CARDIF, IPS1, or VISA) and subsequent downstream activation of intracellular antiviral signaling $(57,59,60)$ (Figure 3). K172 seems to play a central role in this process, since the $\mathrm{K} 172 \mathrm{R}$ mutation severely reduces ubiquitination of the CARD domain of RIG-I and subsequent binding to MAVS $(57,59)$. Interestingly, phosphorylation of the CARD domains of RIG-I prevents CARD ubiquitination, indicating that phosphatases need to first dephosphorylate CARD-RIG-I and allow TRIM25-mediated polyubiquitination (61-64). In addition to anchored ubiquitin, RIG-I can be activated by binding through its CARD domains to unanchored ubiquitin chains assembled by TRIM25 (30). Following RIG-I activation, TRIM25-mediated K48-linked ubiquitination and subsequent proteasomal degradation of the larger MAVS isoform seems to be required for the downstream signaling that leads to type I IFN (65), although other studies have shown opposite results (66) (Figure 3). Studies in mouse embryonic fibroblasts deficient in TRIM25 demonstrated its importance in RIG-I activation and IFN- $\beta$ production in response to viral infection (57). All those experiments demonstrated the essential role of TRIM25 in RIG-I activation and signaling. Nevertheless, the RIPLET E3 ligase has also been shown to be involved in RIG-I polyubiquitination and sustained activation $(67,68)$. RIPLET, such as TRIM25, has an N-terminal RING domain and a C-terminal SPRY domain. However, RIPLET lacks B-box domains and does not belong to the TRIM family. Although it has been reported that RIPLET can mediate K63 polyubiquitination of K172 in the CARD domain of RIG-I (67), it does not seem that TRIM25 and RIPLET play a redundant role in the activation of RIG-I, since RIPLET mainly generates K63 ubiquitin chains in the RIG-I C-terminal regulatory domain $(68,69)$. Some redundancy in RIG-I activation by different TRIM proteins may, however, exist. For example, TRIM4, which belong to the same subgroup as TRIM25 in the TRIM protein family and share with it similar structural characteristics, can also target RIG-I for K63 polyubiquitination in K154, K164, and K172 of its CARD domain, which results in the activation IRF3 and NF- $\kappa \mathrm{B}$, and IFN- $\beta$ production (70).

TRIM25 may, however, have a dual role in RIG-I regulation, as it has been recently reported that TRIM25 negatively regulates RIG-I through stabilization of the ubiquitin-like protein FAT10. FAT10 non-covalently binds to RIG-I and sequesters it from the signaling platform inhibiting IRF3 and NF- $\mathrm{\kappa B}$ activation (71) (Figure 3). This mechanism may contribute to limit the inflammatory response and reduce host damage, since FAT10 is accumulated subsequently to production of inflammatory cytokines late during virus infections (71).

TRIM25 also functions as an E3 ligase to conjugate the ubiquitin-like protein ISG15 to target proteins in a process termed ISGylation (72). Furthermore, auto-ISGylation of TRIM25 negatively regulates its ISG15 E3 ligase activity (73) (Figure 3). 


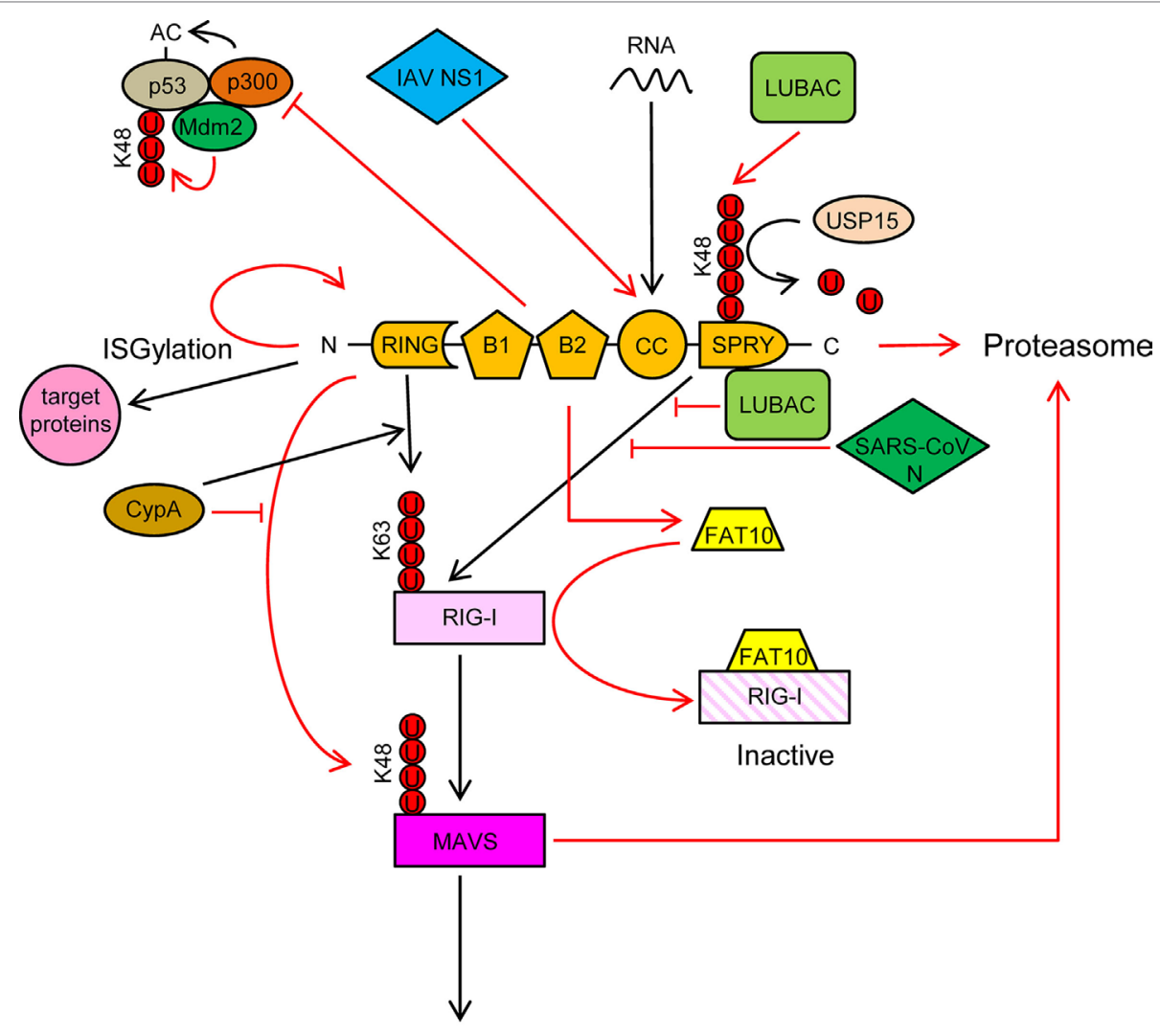

Antiviral response

FIGURE 3 | TRIM25 participates in several innate immune-related processes and its activity is regulated by cellular and viral proteins. See text for detailed description of the individual processes. Ubiquitin molecules are represented by a $U$ inside a red circle. Interactions and inhibitory processes are represented by red lines. The number of Us does not represent the actual level of ubiquitination.

More than 300 proteins, both cellular and pathogen-encoded, have been identified as targets for ISG15 conjugation (74). In general, ISGylation, as well as free ISG15, has broad-spectrum antiviral effects (75-83), although a pro-viral effect has been described in the case of hepatitis $C$ virus (84-86). Furthermore, human ISG15 has been shown to stabilize USP18, a negative regulator of the type I IFN receptor (87). In addition, it has been reported that some viruses have developed mechanisms to counteract the antiviral actions of ISG15, reflecting the importance of this molecule in the response against virus infections $(88,89)$.

In addition to RIG-I, TRIM25 also positively regulates the melanoma differentiation-associated protein 5 (MDA5)-MAVSTRAF6 antiviral axis leading to activation of NF- $\mathrm{B}$ (90). Also, it has been recently reported that the antiviral action of zing-finger antiviral protein (ZAP), a cellular protein that inhibits viral mRNAs translation, is enhanced by interaction with the SPRY domain of TRIM25 $(91,92)$.

TRIM25 may also associate with p53, and it has been proposed that this association promotes p53 degradation, since TRIM25 silencing increased the accumulation of p53 and reduced proliferation and migration of lung cancer cells (93). By contrast, other study showed that TRIM25 enhanced p53 levels by preventing their ubiquitination and proteasomal degradation (94). Despite increasing p53 levels, TRIM25 also inhibited p53 activity by preventing its acetylation by $\mathrm{p} 300$, an essential modification for the transcriptional activation of p53 target genes (Figure 3) (94). In any case, the regulation of p53 levels and/or activity by TRIM25 may have a deep impact on the innate immune response against infecting pathogens, as it has been reported that p53 upregulates the expression of interferon-stimulated genes (ISGs) either directly or through upregulation of IRF9, a component of the ISG factor 3 (ISGF3) (95-100).

An interesting observation is that TRIM25 can bind RNA through its central CC domain (101-103) (Figure 3). This opens new possibilities by which TRIM25 may influence intracellular signaling and/or replication of RNA viruses. It has been proposed that TRIM25 may use the RNA as a scaffold to get close to and modify its targets, including RIG-I and viral ribonucleoproteins (56). By contrast, as described below, some RNA viruses may take advantage of the capacity of TRIM25 to bind RNA to inhibit its function (104).

In conclusion, it is becoming evident that TRIM25 acts on multiple steps of signaling pathways inside the cell. Both positive and negative regulations of these pathways have been reported, adding complexity and relevance to the role of TRIM25 in the 
intracellular innate response. Pieces of evidence of the physiological relevance of TRIM25 in vivo, however, are indirect. For example, TRIM25 polymorphisms have been associated with differences in the humoral response and secretion of some cytokines following measles virus vaccination in children (105); and, as described below, particular virus infections of mice also suggest a role of TRIM25 in regulating RIG-I in vivo $(66,106)$. Finally, the fact that TRIM25 has been under positive selection pressure in primates $(107,108)$ and that it is a targeted by some viral proteins (see next section) also indicates an important role of this molecule in the in vivo immunity against viruses.

\section{TRIM25 ACTIVITY IS CONTROLLED BY CELLULAR AND VIRAL PROTEINS}

A strong innate immune response, including RIG-I-mediated signaling, is necessary for virus clearance but it has to be tightly regulated to prevent excessive inflammation. Therefore, it is not surprising that immune signaling pathways are regulated by numerous positive and negative interactions, many of which are targeted by virus proteins in order to facilitate virus replication.

Cyclophilin A (CypA), a peptidyl-prolyl cis/trans isomerase, positively regulates RIG-I signaling by two different mechanisms (66): (a) CypA promotes the interaction between RIG-I and TRIM25, which results in increased TRIM25-mediated K63linked ubiquitination and activation of RIG-I (Figure 3) and (b) CypA competes with TRIM25 for the interaction with MAVS, which reduces TRIM25-induced K48-linked ubiquitination and proteasomal degradation of MAVS (Figure 3). Accordingly, infection of CypA knockout $(\mathrm{KO})$ mice with Sendai virus resulted in reduced expression of type I IFNs and ISGs, higher viral load, and severer histopathology in lungs, as compared with wild-type infections (66). However, it remains to be elucidated whether these in vivo effects in $\mathrm{KO}$ mice are mediated by the lack of the CypA-dependent regulation of TRIM25 activity or by a different mechanism.

The linear ubiquitination assembly complex (LUBAC), composed of the E3 ligases heme-oxidized IRP2 ubiquitin ligase-1 (HOIL-1L) and HOIL-1-interacting protein (HOIP), negatively regulates RIG-I signaling using two different mechanisms (109): (a) LUBAC ubiquitinates the C-terminal SPRY domain of TRIM25, leading to TRIM25 degradation by the proteasome (Figure 3). TRIM25 ubiquitination depends on the RBR (RING-IBR-RING) domains of both HOIL-1L and HOIP; (b) The Npl4-type zinc finger (NZF) domain of HOIL-1L competes with TRIM25 for RIG-I interaction, thereby blocking ubiquitination and activation of RIG-I by TRIM25 (109) (Figure 3). Recently, the ubiquitin-specific protease 15 (USP15), a TRIM25-interacting protein, was reported to neutralize the inhibitory effect of LUBAC (110). USP15 binds to TRIM25 in viral infections, detaching the K48-linked ubiquitin chains assembled by LUBAC on TRIM25, thereby stabilizing the TRIM25 protein levels and promoting a sustained antiviral response (Figure 3).

TRK-fused gene (TFG) protein, which is another protein that interacts with TRIM25, has also been reported to inhibit the antiviral signaling mediated by RIG-I, although it is not sure that the interaction between TFG and TRIM25 itself is imperative for that effect (111).

It has been reported that some viral proteins are able to interact with TRIM25 and inhibit RIG-I activation. For example, the non-structural protein 1 (NS1) of influenza A virus (IAV) interacts with the CC domain of TRIM25 preventing its dimerization and K63-linked ubiquitination of the RIG-I CARDs, thereby suppressing RIG-I signal transduction $(60,106,112)$ (Figure 3). This interaction seems to be mediated by residues E96/E97 in NS1, since an E96A/E97A NS1 mutant cannot interact with TRIM25, and also did not inhibit ubiquitinationmediated RIG-I signaling (106). Interestingly, in contrast to the wild-type virus, the E96A/E97A NS1 mutant was not virulent in mice (106). Since residues 96 and 97 have not been associated with other NS1 functions, it is tempting to speculate that the interaction between TRIM25 and NS1 is required for IAV virulence. In addition to its role in inhibiting RIG-I activation, the TRIM25-binding domain of IAV NS1 is needed for suppression of IL- $1 \beta$ secretion mediated by NLRP3 in macrophages, suggesting that TRIM25 may also be involved in the activation of this pathway $(113,114)$.

Another example of TRIM25 regulation by viral proteins is the $\mathrm{N}$ proteins of the severe acute respiratory syndrome coronavirus (SARS-CoV), which binds to the SPRY domain of TRIM25, thereby inhibiting activation of RIG-I by TRIM25 ubiquitination (115) (Figure 3).

Finally, it has been recently reported that subgenomic RNA from dengue virus binds TRIM25, preventing its USP15dependent deubiquitination necessary for efficient RIG-I activation (104).

\section{CONCLUDING REMARKS}

Emerging data show that the mechanisms by which TRIM25 may modulate the innate immune response against viruses are multiple and more complex than previously thought. TRIM25 has a dual role in RIG-I regulation: while TRIM25-mediated ubiquitination of RIG-I is essential for the transmission of downstream signaling, TRIM25 stabilization of FAT10 blocks active RIG-I. In addition, TRIM25 inhibits p53 acetylation, a modification indispensable for p53 antiviral activity. Finally, the ability of TRIM25 to bind RNA suggests new lines of investigation to uncover additional mechanisms of action and potential targets of this molecule. This complexity also increases the opportunities to develop novel strategies to regulate the innate immune response in order to reduce viral replication and/or avoid undesired excessive inflammation.

\section{AUTHOR CONTRIBUTIONS}

MM-V: investigation, resources and data curation, visualization, and writing-original draft preparation. SR: funding acquisition, writing-review, and editing. LM and AG-S: writing-review and editing. IM: conceptualization, funding acquisition, investigation, 
resources and data curation, project administration, supervision, visualization, writing-original draft preparation, writing-review, and editing.

\section{FUNDING}

We gratefully acknowledge financial support from Instituto de Salud Carlos III, grant numbers PI15CIII/00024 (IM),

\section{REFERENCES}

1. Broz P, Monack DM. Newly described pattern recognition receptors team up against intracellular pathogens. Nat Rev Immunol (2013) 13:551-65. doi:10.1038/nri3479

2. Creagh EM, O'Neill LA. TLRs, NLRs and RLRs: a trinity of pathogen sensors that co-operate in innate immunity. Trends Immunol (2006) 27:352-7. doi:10.1016/j.it.2006.06.003

3. Takeuchi O, Akira S. Pattern recognition receptors and inflammation. Cell (2010) 140:805-20. doi:10.1016/j.cell.2010.01.022

4. Sun L, Liu S, Chen ZJ. SnapShot: pathways of antiviral innate immunity. Cell (2010) 140(436-436):e432. doi:10.1016/j.cell.2010.01.041

5. Pham AM, Tenoever BR. The IKK kinases: operators of antiviral signaling. Viruses (2010) 2:55-72. doi:10.3390/v2010055

6. Sharma S, tenOever BR, Grandvaux N, Zhou GP, Lin R, Hiscott J. Triggering the interferon antiviral response through an IKK-related pathway. Science (2003) 300:1148-51. doi:10.1126/science.1081315

7. Kumar H, Kawai T, Akira S. Pathogen recognition by the innate immune system. Int Rev Immunol (2011) 30:16-34. doi:10.3109/08830185.2010.529976

8. Sen GC, Sarkar SN. The interferon-stimulated genes: targets of direct signaling by interferons, double-stranded RNA, and viruses. Curr Top Microbiol Immunol (2007) 316:233-50. doi:10.1007/978-3-540-71329-6_12

9. Jefferies C, Wynne C, Higgs R. Antiviral TRIMs: friend or foe in autoimmune and autoinflammatory disease? Nat Rev Immunol (2011) 11:617-25. doi:10.1038/nri3043

10. Kato H, Fujita T. Autoimmunity caused by constitutive activation of cytoplasmic viral RNA sensors. Cytokine Growth Factor Rev (2014) 25:739-43. doi:10.1016/j.cytogfr.2014.08.003

11. Smith S, Jefferies C. Role of DNA/RNA sensors and contribution to autoimmunity. Cytokine Growth Factor Rev (2014) 25:745-57. doi:10.1016/ j.cytogfr.2014.07.019

12. Davis ME, Gack MU. Ubiquitination in the antiviral immune response. Virology (2015) 47(9-480):52-65. doi:10.1016/j.virol.2015.02.033

13. Harhaj EW, Dixit VM. Deubiquitinases in the regulation of NF-kappaB signaling. Cell Res (2011) 21:22-39. doi:10.1038/cr.2010.166

14. Maelfait J, Beyaert R. Emerging role of ubiquitination in antiviral RIG-I signaling. Microbiol Mol Biol Rev (2012) 76:33-45. doi:10.1128/MMBR. 05012-11

15. Oshiumi H, Matsumoto M, Seya T. Ubiquitin-mediated modulation of the cytoplasmic viral RNA sensor RIG-I. J Biochem (2012) 151:5-11. doi:10.1093/ $\mathrm{jb} / \mathrm{mvr} 111$

16. Zinngrebe J, Montinaro A, Peltzer N, Walczak H. Ubiquitin in the immune system. EMBO Rep (2014) 15:28-45. doi:10.1002/embr.201338025

17. Baker RT, Board PG. The human ubiquitin gene family: structure of a gene and pseudogenes from the Ub B subfamily. Nucleic Acids Res (1987) 15:443-63. doi:10.1093/nar/15.2.443

18. Ciechanover A, Elias S, Heller H, Hershko A. "Covalent affinity" purification of ubiquitin-activating enzyme. J Biol Chem (1982) 257:2537-42.

19. Hershko A, Heller H, Elias S, Ciechanover A. Components of ubiquitin-protein ligase system. Resolution, affinity purification, and role in protein breakdown. J Biol Chem (1983) 258:8206-14.

20. Kerscher O, Felberbaum R, Hochstrasser M. Modification of proteins by ubiquitin and ubiquitin-like proteins. Annu Rev Cell Dev Biol (2006) 22:159-80. doi:10.1146/annurev.cellbio.22.010605.093503

21. Ye Y, Rape M. Building ubiquitin chains: E2 enzymes at work. Nat Rev Mol Cell Biol (2009) 10:755-64. doi:10.1038/nrm2780
PI14CIII/00011 (SR), and CD14/00002 (LM). AG-S laboratories are partly supported by NIH grants R33AI119304, R21AI129486, U19AI106754, P01AI097092, R01DA033773, R01AI088770, U19AI117873, U19AI118610, R01AI125524, U01AI124297, and R01AI127658, contract HHSN272201300023C and by CRIP (Center for Research on Influenza Pathogenesis), an NIAID funded Center of Excellence for Influenza Surveillance and Research (CEIRS, contract HHSN272201400008C).

22. Kirisako T, Kamei K, Murata S, Kato M, Fukumoto H, Kanie M, et al. A ubiq uitin ligase complex assembles linear polyubiquitin chains. EMBO J (2006) 25:4877-87. doi:10.1038/sj.emboj.7601360

23. Iwai K, Fujita H, Sasaki Y. Linear ubiquitin chains: NF-kappaB signalling, cell death and beyond. Nat Rev Mol Cell Biol (2014) 15:503-8. doi:10.1038/ nrm3836

24. Tokunaga F, Iwai K. LUBAC, a novel ubiquitin ligase for linear ubiquitination, is crucial for inflammation and immune responses. Microbes Infect (2012) 14:563-72. doi:10.1016/j.micinf.2012.01.011

25. Kulathu Y, Komander D. Atypical ubiquitylation - the unexplored world of polyubiquitin beyond Lys48 and Lys63 linkages. Nat Rev Mol Cell Biol (2012) 13:508-23. doi:10.1038/nrm3394

26. Dikic I, Wakatsuki S, Walters KJ. Ubiquitin-binding domains - from structures to functions. Nat Rev Mol Cell Biol (2009) 10:659-71. doi:10.1038/nrm2767

27. Hicke L, Schubert HL, Hill CP. Ubiquitin-binding domains. Nat Rev Mol Cell Biol (2005) 6:610-21. doi:10.1038/nrm1701

28. Hurley JH, Lee S, Prag G. Ubiquitin-binding domains. Biochem J (2006) 399:361-72. doi:10.1042/BJ20061138

29. Rajsbaum R, Versteeg GA, Schmid S, Maestre AM, Belicha-Villanueva A, Martinez-Romero C, et al. Unanchored K48-linked polyubiquitin synthesized by the E3-ubiquitin ligase TRIM6 stimulates the interferon-IKKepsilon kinase-mediated antiviral response. Immunity (2014) 40:880-95. doi:10.1016/j.immuni.2014.04.018

30. Zeng W, Sun L, Jiang X, Chen X, Hou F, Adhikari A, et al. Reconstitution of the RIG-I pathway reveals a signaling role of unanchored polyubiquitin chains in innate immunity. Cell (2010) 141:315-30. doi:10.1016/j.cell. 2010.03.029

31. Joazeiro CA, Weissman AM. RING finger proteins: mediators of ubiquitin ligase activity. Cell (2000) 102:549-52. doi:10.1016/S0092-8674(00)00077-5

32. Meroni G, Diez-Roux G. TRIM/RBCC, a novel class of 'single protein RING finger' E3 ubiquitin ligases. Bioessays (2005) 27:1147-57. doi:10.1002/ bies. 20304

33. Berndsen CE, Wolberger C. New insights into ubiquitin E3 ligase mechanism. Nat Struct Mol Biol (2014) 21:301-7. doi:10.1038/nsmb.2780

34. Kentsis A, Borden KL. Construction of macromolecular assemblages in eukaryotic processes and their role in human disease: linking RINGs together. Curr Protein Pept Sci (2000) 1:49-73. doi:10.2174/1389203003381478

35. Metzger MB, Pruneda JN, Klevit RE, Weissman AM. RING-type E3 ligases: master manipulators of E2 ubiquitin-conjugating enzymes and ubiquitination. Biochim Biophys Acta (2014) 1843:47-60. doi:10.1016/j.bbamcr.2013.05.026

36. Massiah MA, Matts JA, Short KM, Simmons BN, Singireddy S, Yi Z, et al. Solution structure of the MID1 B-box2 CHC(D/C)C(2)H(2) zinc-binding domain: insights into an evolutionarily conserved RING fold. J Mol Biol (2007) 369:1-10. doi:10.1016/j.jmb.2007.03.017

37. Massiah MA, Simmons BN, Short KM, Cox TC. Solution structure of the RBCC/TRIM B-box1 domain of human MID1: B-box with a RING. J Mol Biol (2006) 358:532-45. doi:10.1016/j.jmb.2006.02.009

38. Diaz-Griffero F, Qin XR, Hayashi F, Kigawa T, Finzi A, Sarnak Z, et al. A B-box 2 surface patch important for TRIM5alpha self-association, capsid binding avidity, and retrovirus restriction. J Virol (2009) 83:10737-51. doi:10.1128/ JVI.01307-09

39. Li X, Yeung DF, Fiegen AM, Sodroski J. Determinants of the higher order association of the restriction factor TRIM5alpha and other tripartite motif (TRIM) proteins. J Biol Chem (2011) 286:27959-70. doi:10.1074/jbc.M111.260406

40. Tao H, Simmons BN, Singireddy S, Jakkidi M, Short KM, Cox TC, et al. Structure of the MID1 tandem B-boxes reveals an interaction reminiscent 
of intermolecular ring heterodimers. Biochemistry (2008) 47:2450-7. doi:10.1021/bi7018496

41. Streich FC Jr, Ronchi VP, Connick JP, Haas AL. Tripartite motif ligases catalyze polyubiquitin chain formation through a cooperative allosteric mechanism. J Biol Chem (2013) 288:8209-21. doi:10.1074/jbc.M113.451567

42. D'Cruz AA, Babon JJ, Norton RS, Nicola NA, Nicholson SE. Structure and function of the SPRY/B30.2 domain proteins involved in innate immunity. Protein Sci (2013) 22:1-10. doi:10.1002/pro.2185

43. Hatakeyama S. TRIM proteins and cancer. Nat Rev Cancer (2011) 11:792-804. doi:10.1038/nrc3139

44. Napolitano LM, Meroni G. TRIM family: pleiotropy and diversification through homomultimer and heteromultimer formation. IUBMB Life (2012) 64:64-71. doi:10.1002/iub.580

45. Rajsbaum R, Garcia-Sastre A, Versteeg GA. TRIMmunity: the roles of the TRIM E3-ubiquitin ligase family in innate antiviral immunity. J Mol Biol (2014) 426:1265-84. doi:10.1016/j.jmb.2013.12.005

46. Kawai T, Akira S. Regulation of innate immune signalling pathways by the tripartite motif (TRIM) family proteins. EMBO Mol Med (2011) 3:513-27. doi:10.1002/emmm.201100160

47. Medrano LM, Rallon N, Berenguer J, Jimenez-Sousa MA, Soriano V, AldamizEchevarria T, et al. Relationship of TRIM5 and TRIM22 polymorphisms with liver disease and HCV clearance after antiviral therapy in HIV/HCV coinfected patients. J Transl Med (2016) 14:257. doi:10.1186/s12967-016-1005-7

48. Nisole S, Stoye JP, Saib A. TRIM family proteins: retroviral restriction and antiviral defence. Nat Rev Microbiol (2005) 3:799-808. doi:10.1038/nrmicro1248

49. Ozato K, Shin DM, Chang TH, Morse HC III. TRIM family proteins and their emerging roles in innate immunity. Nat Rev Immunol (2008) 8:849-60. doi:10.1038/nri2413

50. Santa-Marta M, de Brito PM, Godinho-Santos A, Goncalves J. Host factors and HIV-1 replication: clinical evidence and potential therapeutic approaches. Front Immunol (2013) 4:343. doi:10.3389/fimmu.2013.00343

51. Uchil PD, Hinz A, Siegel S, Coenen-Stass A, Pertel T, Luban J, et al. TRIM protein-mediated regulation of inflammatory and innate immune signaling and its association with antiretroviral activity. J Virol (2013) 87:257-72. doi:10.1128/JVI.01804-12

52. Versteeg GA, Rajsbaum R, Sanchez-Aparicio MT, Maestre AM, Valdiviezo J, Shi $M$, et al. The E3-ligase TRIM family of proteins regulates signaling pathways triggered by innate immune pattern-recognition receptors. Immunity (2013) 38:384-98. doi:10.1016/j.immuni.2012.11.013

53. Yang C, Zhao X, Sun D, Yang L, Chong C, Pan Y, et al. Interferon alpha (IFNalpha)-induced TRIM22 interrupts HCV replication by ubiquitinating NS5A. Cell Mol Immunol (2016) 13:94-102. doi:10.1038/cmi.2014.131

54. Carthagena L, Bergamaschi A, Luna JM, David A, Uchil PD, MargottinGoguet F, et al. Human TRIM gene expression in response to interferons. PLoS One (2009) 4:e4894. doi:10.1371/journal.pone.0004894

55. Inoue S, Orimo A, Hosoi T, Kondo S, Toyoshima H, Kondo T, et al. Genomic binding-site cloning reveals an estrogen-responsive gene that encodes a RING finger protein. Proc Natl Acad Sci U S A (1993) 90:11117-21. doi:10.1073/ pnas.90.23.11117

56. Heikel G, Choudhury NR, Michlewski G. The role of Trim25 in development, disease and RNA metabolism. Biochem Soc Trans (2016) 44:1045-50. doi:10.1042/BST20160077

57. Gack MU, Shin YC, Joo CH, Urano T, Liang C, Sun L, et al. TRIM25 RINGfinger E3 ubiquitin ligase is essential for RIG-I-mediated antiviral activity. Nature (2007) 446:916-20. doi:10.1038/nature05732

58. D'Cruz AA, Kershaw NJ, Chiang JJ, Wang MK, Nicola NA, Babon JJ, et al. Crystal structure of the TRIM25 B30.2 (PRYSPRY) domain: a key component of antiviral signalling. Biochem J (2013) 456:231-40. doi:10.1042/BJ20121425

59. Gack MU, Kirchhofer A, Shin YC, Inn KS, Liang C, Cui S, et al. Roles of RIG-I N-terminal tandem CARD and splice variant in TRIM25-mediated antiviral signal transduction. Proc Natl Acad Sci U S A (2008) 105:16743-8. doi:10.1073/pnas.0804947105

60. Rajsbaum R, Albrecht RA, Wang MK, Maharaj NP, Versteeg GA, Nistal-Villan E, et al. Species-specific inhibition of RIG-I ubiquitination and IFN induction by the influenza A virus NS1 protein. PLoS Pathog (2012) 8:e1003059. doi:10.1371/journal.ppat.1003059

61. Gack MU, Nistal-Villan E, Inn KS, Garcia-Sastre A, Jung JU. Phosphorylationmediated negative regulation of RIG-I antiviral activity. J Virol (2010) 84:3220-9. doi:10.1128/JVI.02241-09
62. Maharaj NP, Wies E, Stoll A, Gack MU. Conventional protein kinase C-alpha (PKC-alpha) and PKC-beta negatively regulate RIG-I antiviral signal transduction. J Virol (2012) 86:1358-71. doi:10.1128/JVI.06543-11

63. Nistal-Villan E, Gack MU, Martinez-Delgado G, Maharaj NP, Inn KS, Yang $\mathrm{H}$, et al. Negative role of RIG-I serine 8 phosphorylation in the regulation of interferon-beta production. J Biol Chem (2010) 285:20252-61. doi:10.1074/ jbc.M109.089912

64. Wies E, Wang MK, Maharaj NP, Chen K, Zhou S, Finberg RW, et al. Dephosphorylation of the RNA sensors RIG-I and MDA5 by the phosphatase PP1 is essential for innate immune signaling. Immunity (2013) 38:437-49. doi:10.1016/j.immuni.2012.11.018

65. Castanier C, Zemirli N, Portier A, Garcin D, Bidere N, Vazquez A, et al. MAVS ubiquitination by the E3 ligase TRIM25 and degradation by the proteasome is involved in type I interferon production after activation of the antiviral RIG-I-like receptors. BMC Biol (2012) 10:44. doi:10.1186/1741-7007-10-44

66. Liu W, Li J, Zheng W, Shang Y, Zhao Z, Wang S, et al. Cyclophilin A-regulated ubiquitination is critical for RIG-I-mediated antiviral immune responses. Elife (2017) 6:e24425. doi:10.7554/eLife.24425

67. Gao D, Yang YK, Wang RP, Zhou X, Diao FC, Li MD, et al. REUL is a novel E3 ubiquitin ligase and stimulator of retinoic-acid-inducible gene-I. PLoS One (2009) 4:e5760. doi:10.1371/journal.pone.0005760

68. Oshiumi H, Matsumoto M, Hatakeyama S, Seya T. Riplet/RNF135, a RING finger protein, ubiquitinates RIG-I to promote interferon-beta induction during the early phase of viral infection. J Biol Chem (2009) 284:807-17. doi:10.1074/jbc.M804259200

69. Oshiumi H, Miyashita M, Inoue N, Okabe M, Matsumoto M, Seya T. The ubiquitin ligase Riplet is essential for RIG-I-dependent innate immune responses to RNA virus infection. Cell Host Microbe (2010) 8:496-509. doi:10.1016/ j.chom.2010.11.008

70. Yan J, Li Q, Mao AP, Hu MM, Shu HB. TRIM4 modulates type I interferon induction and cellular antiviral response by targeting RIG-I for K63-linked ubiquitination. J Mol Cell Biol (2014) 6:154-63. doi:10.1093/jmcb/mju005

71. Nguyen NT, Now H, Kim WJ, Kim N, Yoo JY. Ubiquitin-like modifier FAT10 attenuates RIG-I mediated antiviral signaling by segregating activated RIG-I from its signaling platform. Sci Rep (2016) 6:23377. doi:10.1038/srep23377

72. Zou W, Zhang DE. The interferon-inducible ubiquitin-protein isopeptide ligase (E3) EFP also functions as an ISG15 E3 ligase. J Biol Chem (2006) 281:3989-94. doi:10.1074/jbc.M510787200

73. Zou W, Wang J, Zhang DE. Negative regulation of ISG15 E3 ligase EFP through its autoISGylation. Biochem Biophys Res Commun (2007) 354:321-7. doi:10.1016/j.bbrc.2006.12.210

74. Durfee LA, Lyon N, Seo K, Huibregtse JM. The ISG15 conjugation system broadly targets newly synthesized proteins: implications for the antiviral function of ISG15. Mol Cell (2010) 38:722-32. doi:10.1016/j.molcel.2010.05.002

75. Gonzalez-Sanz R, Mata M, Bermejo-Martin J, Alvarez A, Cortijo J, Melero JA, et al. ISG15 is upregulated in respiratory syncytial virus infection and reduces virus growth through protein ISGylation. J Virol (2016) 90:3428-38. doi:10.1128/JVI.02695-15

76. Guerra S, Caceres A, Knobeloch KP, Horak I, Esteban M. Vaccinia virus E3 protein prevents the antiviral action of ISG15. PLoS Pathog (2008) 4:e1000096. doi:10.1371/journal.ppat.1000096

77. Hishiki T, Han Q, Arimoto K, Shimotohno K, Igarashi T, Vasudevan SG, et al. Interferon-mediated ISG15 conjugation restricts dengue virus 2 replication. Biochem Biophys Res Commun (2014) 448:95-100. doi:10.1016/ j.bbrc.2014.04.081

78. Lenschow DJ, Lai C, Frias-Staheli N, Giannakopoulos NV, Lutz A, Wolff T, et al. IFN-stimulated gene 15 functions as a critical antiviral molecule against influenza, herpes, and Sindbis viruses. Proc Natl Acad Sci U S A (2007) 104:1371-6. doi:10.1073/pnas.0607038104

79. Ma XZ, Bartczak A, Zhang J, He W, Shalev I, Smil D, et al. Protein interferonstimulated gene 15 conjugation delays but does not overcome coronavirus proliferation in a model of fulminant hepatitis. J Virol (2014) 88:6195-204. doi:10.1128/JVI.03801-13

80. Okumura A, Lu G, Pitha-Rowe I, Pitha PM. Innate antiviral response targets HIV-1 release by the induction of ubiquitin-like protein ISG15. Proc Natl Acad Sci U S A (2006) 103:1440-5. doi:10.1073/pnas.0510518103

81. Okumura A, Pitha PM, Harty RN. ISG15 inhibits Ebola VP40 VLP budding in an L-domain-dependent manner by blocking Nedd4 ligase activity. Proc Natl Acad Sci U S A (2008) 105:3974-9. doi:10.1073/pnas.0710629105 
82. Werneke SW, Schilte C, Rohatgi A, Monte KJ, Michault A, ArenzanaSeisdedos F, et al. ISG15 is critical in the control of Chikungunya virus infection independent of UbE1L mediated conjugation. PLoS Pathog (2011) 7: e1002322. doi:10.1371/journal.ppat.1002322

83. Yanguez E, Garcia-Culebras A, Frau A, Llompart C, Knobeloch KP, GutierrezErlandsson S, et al. ISG15 regulates peritoneal macrophages functionality against viral infection. PLoS Pathog (2013) 9:e1003632. doi:10.1371/journal. ppat.1003632

84. Broering R, Zhang X, Kottilil S, Trippler M, Jiang M, Lu M, et al. The interferon stimulated gene 15 functions as a proviral factor for the hepatitis $\mathrm{C}$ virus and as a regulator of the IFN response. Gut (2010) 59:1111-9. doi:10.1136/ gut.2009.195545

85. Chen L, Sun J, Meng L, Heathcote J, Edwards AM, McGilvray ID. ISG15, a ubiquitin-like interferon-stimulated gene, promotes hepatitis $\mathrm{C}$ virus production in vitro: implications for chronic infection and response to treatment. J Gen Virol (2010) 91:382-8. doi:10.1099/vir.0.015388-0

86. Real CI, Megger DA, Sitek B, Jahn-Hofmann K, Ickenstein LM, John MJ, et al. Identification of proteins that mediate the pro-viral functions of the interferon stimulated gene 15 in hepatitis C virus replication. Antiviral Res (2013) 100:654-61. doi:10.1016/j.antiviral.2013.10.009

87. Zhang X, Bogunovic D, Payelle-Brogard B, Francois-Newton V, Speer SD, Yuan C, et al. Human intracellular ISG15 prevents interferon-alpha/beta over-amplification and auto-inflammation. Nature (2015) 517:89-93. doi:10.1038/nature13801

88. Eduardo-Correia B, Martinez-Romero C, Garcia-Sastre A, Guerra S. ISG15 is counteracted by vaccinia virus $\mathrm{E} 3$ protein and controls the proinflammatory response against viral infection. J Virol (2014) 88:2312-8. doi:10.1128/ JVI.03293-13

89. Yuan W, Krug RM. Influenza B virus NS1 protein inhibits conjugation of the interferon (IFN)-induced ubiquitin-like ISG15 protein. EMBO J (2001) 20:362-71. doi:10.1093/emboj/20.3.362

90. Lee NR, Kim HI, Choi MS, Yi CM, Inn KS. Regulation of MDA5-MAVS antiviral signaling axis by TRIM25 through TRAF6-mediated NF-kappaB activation. Mol Cells (2015) 38:759-64. doi:10.14348/molcells.2015.0047

91. Li MM, Lau Z, Cheung P, Aguilar EG, Schneider WM, Bozzacco L, et al. TRIM25 enhances the antiviral action of zinc-finger antiviral protein (ZAP). PLoS Pathog (2017) 13:e1006145. doi:10.1371/journal.ppat.1006145

92. Zheng X, Wang X, Tu F, Wang Q, Fan Z, Gao G. TRIM25 is required for the antiviral activity of zinc finger antiviral protein. J Virol (2017) 91:e88-17. doi:10.1128/JVI.00088-17

93. Qin Y, Cui H, Zhang H. Overexpression of TRIM25 in lung cancer regulates tumor cell progression. Technol Cancer Res Treat (2016) 15:707-15. doi:10.1177/1533034615595903

94. Zhang P, Elabd S, Hammer S, Solozobova V, Yan H, Bartel F, et al. TRIM25 has a dual function in the p53/Mdm2 circuit. Oncogene (2015) 34:5729-38. doi:10.1038/onc.2015.21

95. Hummer BT, Li XL, Hassel BA. Role for p53 in gene induction by double-stranded RNA. J Virol (2001) 75:7774-7. doi:10.1128/JVI.75.16. 7774-7777.2001

96. Mori T, Anazawa Y, Iiizumi M, Fukuda S, Nakamura Y, Arakawa H. Identification of the interferon regulatory factor 5 gene (IRF-5) as a direct target for p53. Oncogene (2002) 21:2914-8. doi:10.1038/sj.onc.1205459

97. Munoz-Fontela C, Macip S, Martinez-Sobrido L, Brown L, Ashour J, GarciaSastre A, et al. Transcriptional role of p53 in interferon-mediated antiviral immunity. J Exp Med (2008) 205:1929-38. doi:10.1084/jem.20080383

98. Rivas C, Aaronson SA, Munoz-Fontela C. Dual role of p53 in innate antiviral immunity. Viruses (2010) 2:298-313. doi:10.3390/v2010298

99. Takaoka A, Hayakawa S, Yanai H, Stoiber D, Negishi H, Kikuchi H, et al. Integration of interferon-alpha/beta signalling to p53 responses in tumour suppression and antiviral defence. Nature (2003) 424:516-23. doi:10.1038/ nature 01850

100. Taura M, Eguma A, Suico MA, Shuto T, Koga T, Komatsu K, et al. p53 regulates toll-like receptor 3 expression and function in human epithelial cell lines. Mol Cell Biol (2008) 28:6557-67. doi:10.1128/MCB.01202-08
101. Beckmann BM, Horos R, Fischer B, Castello A, Eichelbaum K, Alleaume AM, et al. The RNA-binding proteomes from yeast to man harbour conserved enigmRBPs. Nat Commun (2015) 6:10127. doi:10.1038/ncomms10127

102. Castello A, Fischer B, Eichelbaum K, Horos R, Beckmann BM, Strein C, et al. Insights into RNA biology from an atlas of mammalian mRNA-binding proteins. Cell (2012) 149:1393-406. doi:10.1016/j.cell.2012.04.031

103. Kwon SC, Yi H, Eichelbaum K, Fohr S, Fischer B, You KT, et al. The RNAbinding protein repertoire of embryonic stem cells. Nat Struct Mol Biol (2013) 20:1122-30. doi:10.1038/nsmb.2638

104. Manokaran G, Finol E, Wang C, Gunaratne J, Bahl J, Ong EZ, et al. Dengue subgenomic RNA binds TRIM25 to inhibit interferon expression for epidemiological fitness. Science (2015) 350:217-21. doi:10.1126/science.aab3369

105. Ovsyannikova IG, Haralambieva IH, Vierkant RA, O’Byrne MM, Poland GA. Associations between polymorphisms in the antiviral TRIM genes and measles vaccine immunity. Hum Immunol (2013) 74:768-74. doi:10.1016/ j.humimm.2013.01.031

106. Gack MU, Albrecht RA, Urano T, Inn KS, Huang IC, Carnero E, et al. Influenza A virus NS1 targets the ubiquitin ligase TRIM25 to evade recognition by the host viral RNA sensor RIG-I. Cell Host Microbe (2009) 5:439-49. doi:10.1016/j.chom.2009.04.006

107. Han K, Lou DI, Sawyer SL. Identification of a genomic reservoir for new TRIM genes in primate genomes. PLoS Genet (2011) 7:e1002388. doi:10.1371/ journal.pgen. 1002388

108. Malfavon-Borja R, Sawyer SL, Wu LI, Emerman M, Malik HS. An evolutionary screen highlights canonical and noncanonical candidate antiviral genes within the primate TRIM gene family. Genome Biol Evol (2013) 5:2141-54. doi:10.1093/gbe/evt163

109. Inn KS, Gack MU, Tokunaga F, Shi M, Wong LY, Iwai K, et al. Linear ubiquitin assembly complex negatively regulates RIG-I- and TRIM25-mediated type I interferon induction. Mol Cell (2011) 41:354-65. doi:10.1016/ j.molcel.2010.12.029

110. Pauli EK, Chan YK, Davis ME, Gableske S, Wang MK, Feister KF, et al. The ubiquitin-specific protease USP15 promotes RIG-I-mediated antiviral signaling by deubiquitylating TRIM25. Sci Signal (2014) 7:ra3. doi:10.1126/ scisignal.2004577

111. Lee NR, Shin HB, Kim HI, Choi MS, Inn KS. Negative regulation of RIGI-mediated antiviral signaling by TRK-fused gene (TFG) protein. Biochem Biophys Res Commun (2013) 437:168-72. doi:10.1016/j.bbrc.2013.06.061

112. Sanchez-Aparicio MT, Ayllon J, Leo-Macias A, Wolff T, Garcia-Sastre A. Subcellular localizations of RIG-I, TRIM25 and MAVS complexes. J Virol (2016) 91:e1155-1116. doi:10.1128/JVI.01155-16

113. Moriyama M, Chen IY, Kawaguchi A, Koshiba T, Nagata K, Takeyama H, et al. The RNA- and TRIM25-binding domains of influenza virus NS1 protein are essential for suppression of NLRP3 inflammasome-mediated interleukin-1beta secretion. J Virol (2016) 90:4105-14. doi:10.1128/JVI.00120-16

114. Pothlichet J, Meunier I, Davis BK, Ting JP, Skamene E, von Messling V, et al. Type I IFN triggers RIG-I/TLR3/NLRP3-dependent inflammasome activation in influenza A virus infected cells. PLoS Pathog (2013) 9:e1003256. doi:10.1371/journal.ppat.1003256

115. Hu Y, Li W, Gao T, Cui Y, Jin Y, Li P, et al. The severe acute respiratory syndrome coronavirus nucleocapsid inhibits type I interferon production by interfering with TRIM25-mediated RIG-I ubiquitination. J Virol (2017) 91: e2143-2116. doi:10.1128/JVI.02143-16

Conflict of Interest Statement: The authors declare that the research was conducted in the absence of any commercial or financial relationships that could be construed as a potential conflict of interest.

Copyright (C) 2017 Martín-Vicente, Medrano, Resino, García-Sastre and Martínez. This is an open-access article distributed under the terms of the Creative Commons Attribution License (CC BY). The use, distribution or reproduction in other forums is permitted, provided the original author(s) or licensor are credited and that the original publication in this journal is cited, in accordance with accepted academic practice. No use, distribution or reproduction is permitted which does not comply with these terms. 